\title{
Additional results on integrals of beam eigenfunctions
}

\section{(遇 Article in press ? )}

- Morales, C.A.Email Author

- View Correspondence (jump link)Universidad Peruana de Ciencias Aplicadas, Facultad de Ingeniería, Lima, Perú

\section{Abstract}

New simplest expression beam mode integrals are presented, including further simplifications and corrections of previous results; this type of integrals appear in computational methods in structural dynamics and vibrations of diverse structures and machines, and in aircraft dynamics methods. (C) 2018, IMechE 2018.

Author keywords

beam eigenfunctionclamped-slidingIntegralsnumerical integrationRayleigh-Ritz

Indexed keywords

Engineering controlled Structural dynamicsVibrations (mechanical) terms:

Compendex keywords Aircraft dynamicsclamped-slidingDynamics and vibrationIntegralsNumerical in Engineering main heading: Eigenvalues and eigenfunctions

- ISSN: 14644193

- Source Type: Journal

- Original language: English

- DOI: $10.1177 / 1464419318761912$

- Document Type: Article in Press

- Publisher: SAGE Publications Ltd 